\title{
Caracterización nutricional y de producción de biomasa de Sambucus peruviana, Sambucus nigra y Morus alba en un banco forrajero
}

\section{Nutritional characterization and production of biomass of Sambucus peruviana, Sambucus nigra and Morus alba in a forage bank}

\author{
Dania Fonseca-López ${ }^{a}$ \\ Anyela Esperanza Salamanca-López, ${ }^{\mathrm{b}}$ \\ Laura Estefanía Niño-Monroy ${ }^{b}$ \\ Carlos Eduardo Rodríguez-Molano ${ }^{\mathrm{b} *}$ \\ José Luis Hoyos-Concha, ${ }^{\mathrm{c}}$ \\ Iván Darío Otero-Ramírez ${ }^{\mathrm{c}}$ \\ Nestor Raúl Torres Lagos ${ }^{\mathrm{d}}$
}

Fecha de Recepción: 26.03.19

Fecha de Aceptación: 08.05.19

DOI: https://doi.org/10.19053/01217488.v10.n2.2019.9098

\section{Resumen}

Los bancos forrajeros son estrategias de cultivo de especies arbustivas en altas densidades de siembra que permiten garantizar la disponibilidad de alimento destinado a rumiantes en las fincas. Por ello, se evaluó la producción de hojas y la calidad nutricional de morera (Morus alba L.), tilo (Sambucus peruviana) y sauco (Sambucus nigra L.) sin fertilización en Boyacá $(2700 \mathrm{msnm}$ ) en un diseño completamente al azar. Se realizó un análisis de varianza (Anova) y donde hubo diferencias significativas se aplicó la prueba de Tukey $(\mathrm{p}<0.05)$. Los resultados fueron, área foliar por planta de $81,82 \pm 0,01 \mathrm{~cm}^{2}, 131,37 \pm 0,01 \mathrm{~cm}^{2}, 89,98 \pm 0,01 \mathrm{~cm}^{2}$; peso de forraje verde individual $1,73 \pm 0,05 \mathrm{~kg}, 2,23 \pm 0,05 \mathrm{~kg}, 1 \pm 0,04 \mathrm{~kg}$; materia seca (MS) $30,8 \pm 0,05 \%$, $20,2 \pm 0,06 \%, 20,1 \pm 0,06 \%$; proteína cruda (PC) 14,62 $\pm 0,01 \%, 14,2 \pm 0,06 \%, 13,4 \pm 0,05 \%$; extracto etéreo (EE) $0,85 \pm 0,01 \%, 1,8 \pm 0,05,1,4 \pm 0,05 \%$; cenizas (CEN) $8,6 \pm 0,05 \%, 1,8 \pm 0,05 \%, 10,07 \pm 0,01 \%$; fibra cruda (FC) $18,97 \pm 0,01 \%, 17,92 \pm 0,18 \%, 17,8 \pm 0,05 \%$; fibra detergente neutra (FDN) $37,8 \pm 0,05 \%, 29,9 \pm 0,05 \%$, $23,1 \pm 0,05 \%$; fibra detergente ácida (FDA) 29,4 $\pm 0,05 \%, 47,3 \pm 0,05 \%, 34,8 \pm 0,05 \%$, para morera, tilo y sauco respectivamente. La biomasa y calidad nutricional varió por especie destacándose tilo con la mayor producción de forraje verde $(6,9$ ton/ha) y proteína de 0,19 ton/ha $(\mathrm{p}<0,05)$.

Palabras clave: forraje, forraje verde, leguminosas forrajeras, nutrientes, plantas de ramoneo, (Agrovoc).

a M.Sc. en Ciencias Veterinarias. Universidad del Cauca. Facultad de Ciencias Agrarias, Grupo de Investigación Nutrición Agropecuaria. (NUTRIFACA). Popayán, Colombia. ORCID: https://orcid.org/0000-0003-3877-3730

b MVZ, Universidad Pedagógica y Tecnológica de Colombia. Facultad de Ciencias Agropecuarias, Grupo de Investigación en Bioquímica Y Nutrición Animal (GIBNA). Tunja, Colombia.

b MVZ, Universidad Pedagógica y Tecnológica de Colombia. Facultad de Ciencias Agropecuarias, Grupo de Investigación en Bioquímica Y Nutrición Animal (GIBNA). Tunja, Colombia. ORCID: https://orcid.org/0000-0002-4056-5351

b *M.Sc. en Ciencias Biológicas. Universidad Pedagógica y Tecnológica de Colombia. Facultad de Ciencias Agropecuarias, Grupo de Investigación en Bioquímica Y Nutrición Animal (GIBNA). Tunja, Colombia. ORCID: https://orcid.org/00000002-0862-3478

c Dr. Ingeniería. Universidad del Cauca. Facultad de Ciencias Agrarias, Grupo de Investigación Aprovechamiento de Subproductos Agroindustriales (ASUBAGROIN). Popayán, Cauca. ORCID: https://orcid.org/0000-0001-9025-9734

c M.C. Microbiología. Universidad del Cauca. Facultad de Ciencias Agrarias, Grupo de Investigación Aprovechamiento de Subproductos Agroindustriales (ASUBAGROIN). Popayán, Cauca,

* Autor de correspondencia: carlos.rodriguez@uptc.edu.co

d M. Sc. en Ciencias Veterinarias. Instructor carrera administrativa. Sena - Regional valle del cauca. Centro agropecuario de Buga 


\begin{abstract}
Forage banks are strategies for cultivating shrub species at high planting densities that guarantee the availability of food for ruminants on farms. Therefore, the production of leaves and the nutritional quality of mulberry (Morus alba L.), tilo (Sambucus peruviana) and sauco (Sambucus nigra L.) without fertilization in Boyacá (2700 masl) were evaluated in a completely random desing. An analysis of variance was performed (Anova) and where there were significant differences the Tukey test was applied $(\mathrm{p}<0.05)$. The results were: leaf area per plant of $81.82 \pm 0.01 \mathrm{~cm} 2,131.37 \pm 0.01 \mathrm{~cm} 2,89.98 \pm 0.01 \mathrm{~cm} 2$; weight of individual green forage $1.73 \pm 0.05 \mathrm{~kg}, 2.23 \pm 0.05 \mathrm{~kg}, 1 \pm 0.04 \mathrm{~kg}$; dry matter (MS) $30.8 \pm 0.05 \%, 20.2 \pm 0.06 \%$, $20.1 \pm 0.06 \%$; crude protein (PC) $14.62 \pm 0.01 \%, 14.2 \pm 0.06 \%, 13.4 \pm 0.05 \%$; Ethereal extract (EE) 0.85 $\pm 0.01 \%, 1.8 \pm 0.05,1.4 \pm 0.05 \%$; ashes (CEN) $8.6 \pm 0.05 \%, 1.8 \pm 0.05 \%, 10.07 \pm 0.01 \%$; crude fiber (FC) $18.97 \pm 0.01 \%, 17.92 \pm 0.18 \%, 17.8 \pm 0.05 \%$; neutral detergent fiber (NDF) $37.8 \pm 0.05 \%, 29.9 \pm 0.05 \%$, $23.1 \pm 0.05 \%$; acid detergent fiber (FDA) $29.4 \pm 0.05 \%, 47.3 \pm 0.05 \%, 34.8 \pm 0.05 \%$, for mulberry, linden and elder respectively. Biomass and nutritional quality varied by species, highlighting lime with the highest production of green fodder $(6.9$ tons / ha) and protein of 0.19 tons / ha $(\mathrm{p}<0.05)$.
\end{abstract}

Key Words: forage, green feed, feed legumes, nutrients, browse plants.

\section{INTRODUCCIÓN}

La alimentación de rumiantes a partir de forrajes sobre los $2500 \mathrm{msnm}$ en el departamento de Boyacá se destaca por especies adaptadas a condiciones de clima frío como falsa poa (Holcus lanatus L.), tréboles (Trifolium spp.), kikuyo (Pennisetum clandestinum Hochst) y el raygrass (Lolium multiflorum Lam) que se ven influenciados por el déficit hídrico y el efecto de las heladas, condiciones que afectan el valor nutritivo del forraje y disponibilidad de biomasa [1], lo que genera sistemas de producción ganaderos tradicionales con bajo nivel de productividad y sostenibilidad [2].

A pesar que los rumiantes pueden aprovechar este tipo de alimento en etapas tardías y baja calidad nutricional, en la mayoría de los casos los forrajes no alcanzan a llenar los requerimientos de los animales teniendo en cuenta que consumen el $10 \%$ de su peso vivo y esta cantidad debe aportar la proteína, grasa, minerales y fibra suficiente para su mantenimiento y producción [2].Lo anterior obliga a buscar alternativas forrajeras sembrados en diferentes sistemas como los bancos de proteína que se basan en la siembra en alta densidad de plantas arbustivas junto con leguminosas lo que permite la obtención en forraje de alta calidad durante las épocas críticas del año [3].

En el departamento de Boyacá se encuentran especies como el sauco, el tilo y la morera que poseen calidad y degradabilidad ruminal aceptables y pueden conservarse en forma de harina y ensilaje [4] [5]. Sin embargo, las variables climáticas repercuten en su adaptación ya que influyen en la riqueza y diversidad de las especies [6]. Además, la calidad nutricional de los forrajes y la respuesta agronómica pueden variar de acuerdo a las características del suelo y la fertilización, donde esta última es una actividad generadora de gases efecto invernadero de modo que la agricultura orgánica que parte del principio de recirculación de nutrientes no solo como compostajes [7] sino mediante los ciclos que se desarrollan con naturalidad como la fijación de nitrógeno en los diferentes ecosistemas es deseable. El objetivo de esta investigación fue evaluar la calidad nutricional y producción de hojas de tilo, sauco y morera en un banco forrajero sin fertilización simulando las condiciones de cultivo tradicionales para generar información aplicable en la región.

\section{MATERIALES Y MÉTODOS}

\subsection{Localización}

El estudio se desarrolló en la granja La María de la Universidad Pedagógica y Tecnológica de Colombia (UPTC) en un cultivo de morera (Morus alba L.), tilo (Sambucus peruviana) y sauco (Sambucus nigra L.) (Clasificación hecha en el Herbario de la UPTC sede Tunja, donde tilo y sauco corresponden a la misma 
especie, variedad diferente aun no avalada por la comunidad científica colombiana según Bravo- $\mathrm{P}$. W.), con poda anual durante tres años establecido en el 2014 en un área de $1061 \mathrm{~m}^{2}$ con una densidad de siembra de $1,20 \mathrm{~m}$ entre surco y $0,8 \mathrm{~m}$ entre planta ubicado en las coordenadas $5^{\circ} 33$ ' 7 " N y $73^{\circ} 21$ ' 23 " W a una altura de $2730 \mathrm{msnm}$ y temperatura media de $21^{\circ} \mathrm{C}$.

\subsection{Diseño experimental}

Las unidades experimentales se tomaron de una población de 195 plantas de morera, 199 de sauco y 13 de tilo en un diseño completamente al azar, y fueron calculadas a partir del siguiente modelo matemático propuesto por Mostacedo y Fredericksen [8].

$$
\mathrm{n}=\frac{\mathrm{t}^{2 *} \mathrm{CV}^{2^{*}}}{\mathrm{E}^{2}+\frac{\mathrm{t}^{2 *} \mathrm{CV}}{\mathrm{N}}}
$$

$\mathrm{n}=$ número de unidades muestrales

$\mathrm{E}=$ error con el que se quiere obtener los valores de un determinado parámetro.

$\mathrm{t}=$ valor que se obtiene de las tablas " $\mathrm{t} " \mathrm{de}$ Student, generalmente se usa $\mathrm{t}=0,05$

$\mathrm{CV}=$ Coeficiente de variación, para obtener este valor es necesario hacer un muestreo piloto.

$\mathrm{N}=$ Tamaño de la población

Se trabajó con un $95 \%$ de confiabilidad según tablas" "t"de Student y con un $20 \%$ de error para las tres especies arbustivas. Se analizaron 21 unidades de morera, 14 de sauco y 7 de tilo.

\subsection{Variables evaluadas}

\section{Análisis composicionales}

Se determinó el contenido de materia seca (MS) con una estufa de secado marca Memmert ${ }^{\circledR}$ (Karl Kolb, Schwabach, Alemania) y humedad (H) por diferencia; cenizas (Cen) por método de incineración a $550^{\circ} \mathrm{C}$ con mufla Thermolyne ${ }^{\circledR}$ (Dubuque, IA) [9]; (\%) proteína cruda por método de KJeldahl, (\%) fibra cruda por método Weende, (\%) extracto etéreo por método Sohxlet, (\%) fibra detergente neutro y (\%) de fibra detergente acida por el método de Van Soest.

\subsection{Producción de forraje verde y área foliar}

Se pesaron las muestras de hojas de cada especie en una balanza y luego se promediaron los valores. El área foliar se calculó con un lector óptico, para ello se tomó al azar una hoja de cada muestra y se estimó su peso, posteriormente se multiplicó por el peso total de hojas de la planta y se dividió por el peso de la hoja con la siguiente fórmula:

$$
\text { Área foliar }=\frac{\text { Área hoja } x \text { peso biomasa individual }}{\text { Peso hoja }}
$$

Para estimar el área foliar total del cultivo se multiplicó el área foliar por planta por el total de arbustos sembrados según especie, relación entre el peso de hojas y área foliar [10].

\subsection{Análisis estadístico}

Los datos se analizaron por un Anova $(\mathrm{p}<0,05)$ y se aplicó la prueba de Tukey donde existió diferencias significativas $(\mathrm{p}<0,05)$. Todos los análisis se hicieron en el paquete estadístico InfoStat.

\section{RESULTADOS Y DISCUSIÓN}

Materia seca (MS). Hubo homogeneidad de varianzas $(p>0,05)$ y diferencias significativas entre el contenido de MS de la morera en comparación con el sauco y el tilo $(p<0,05)$ (Tabla I).En general, la MS de las especies evaluadas fue aceptable pese a no realizarse fertilización desde su establecimiento (20 kg de fertilizante 15-1515 para un área de $1061 \mathrm{~m}^{2} / \mathrm{año}$ ), factor que es determinante en la respuesta forrajera, ya que con aplicaciones de nitrógeno (112,5 kg N/ha/año) se puede duplicar su valor [11]. 
Tabla 1. Resultados composicionales de morera (Morus alba L.), tilo (Sambucus peruviana) y sauco (Sambucus nigra L.)

\begin{tabular}{ccccccccc}
\hline Arbusto & H(\%) & MS(\%) & PC(\%) & EE(\%) & CEN(\%) & FC(\%) & FDN(\%) & FDA(\%) \\
\hline \multirow{3}{*}{ Sauco } & 70,79 & 20,1 & 13,4 & 1,4 & 10,07 & 17,8 & 23,1 & 34,8 \\
& \pm & \pm & \pm & \pm & \pm & \pm & \pm & \pm \\
& $0,01^{\mathrm{b}}$ & $0,06^{\mathrm{b}}$ & $0,05^{\mathrm{c}}$ & $0,05^{\mathrm{b}}$ & $0,01^{\mathrm{a}}$ & $0,05^{\mathrm{b}}$ & $0,05^{\mathrm{c}}$ & $0,05^{\mathrm{b}}$ \\
\multirow{4}{*}{ Morera } & 69,19 & 30,8 & 14,62 & 0,85 & 8,6 & 18,97 & 37,8 & 29,4 \\
& \pm & \pm & \pm & \pm & \pm & \pm & \pm & \pm \\
& $0,01^{\mathrm{c}}$ & $0,05^{\mathrm{a}}$ & $0,01^{\mathrm{a}}$ & $0,01^{\mathrm{c}}$ & $0,05^{\mathrm{b}}$ & $0,01^{\mathrm{a}}$ & $0,05^{\mathrm{a}}$ & $0,05^{\mathrm{c}}$ \\
\multirow{3}{*}{ Tilo } & 78,96 & 20,2 & 14,2 & 1,8 & 1,8 & 17,92 & 29,9 & 47,3 \\
& \pm & \pm & \pm & \pm & \pm & \pm & \pm & \pm \\
& $0,01^{\mathrm{a}}$ & $0,06^{\mathrm{b}}$ & $0,06^{\mathrm{b}}$ & $0,05^{\mathrm{a}}$ & $0,05^{\mathrm{c}}$ & $0,18^{\mathrm{b}}$ & $0,05^{\mathrm{b}}$ & $0,05^{\mathrm{a}}$ \\
\hline
\end{tabular}

H: Humedad, MS: Materia seca, PC: Proteína cruda, EE: Extracto etéreo, CEN: Cenizas, FC: Fibra cruda, FDN: Fibra Detergente Neutra: FDA: Fibra Detergente Ácida. Letras diferentes indican diferencias significativas con un $\mathrm{p}<0,05$ por la prueba de tukey con un $\mathrm{p}$ valor de $0,05 . \pm$ : error estándar.

En sauco es común encontrar MS entre 7,8 (5) y $19,6 \%$ [12] aunque puede alcanzar hasta 22,59 $\%$ [13]. Esto mismo se ha visto en morera con máximo 42,49\% MS en plantas de 2 años [14] $\mathrm{y}$ en tilo $16 \%$ de MS [15]. Esta variabilidad se relaciona con la fase de crecimiento y el nivel de fertilización, pero además, se asocia con: la variedad, la zona, la densidad de siembra, la aplicación de fertilizante, la técnica de cosecha, [9] y la frecuencia de podas, debido a que la planta puede regenerarse y reponer biomasa de manera más lenta con mayor intervalo de tiempo entre cortes durante las primeras semanas por mayor disposición de carbohidratos, rebrotes y hojas que le permiten lograr un óptimo proceso de fotosíntesis, rápida recuperación y producción [10] [16].

\subsection{Humedad (H)}

Los resultados de $\mathrm{H}$ cumplieron con el criterio de homogeneidad de varianzas $(\mathrm{p}<0,05)$ y hubo diferencias significativas $(\mathrm{p}<0,05)$ entre las tres especies arbustivas, donde la mayor media fue para el tilo, seguido del sauco y la morera. El valor hallado para morera, se encuentra entre 65,4 a $80,97 \%$ [11, 17, 18, 19] pero la $\mathrm{H}$ del sauco fue menor (82,2 a 85,2 \%) [20], [5]. Sin embargo, los valores obtenidos para cada uno de los forrajes se encuentran entre el 60 y $70 \%$ de $\mathrm{H}$ que corresponde a un rango óptimo [21].Las variaciones en esta característica se relaciona con la evapotranspiración de las plantas, que puede ser afectada por factores como el tipo de cultivo, variedad, las etapas de desarrollo y las variables climáticas (Radiación solar, temperatura del aire, humedad atmosférica y la velocidad del viento) [22].

\subsection{Proteína cruda (PC}

Hubo diferencias significativas en el contenido de PC de todos los materiales evaluados, donde morera $(14,62 \pm 0,01)$ obtuvo el mayor valor seguido del tilo $(14,2 \pm 0,06)$ y sauco $(13,4 \pm$ $0,05)(p<0,05)$. La PC de morera coincide con un material recolectado y mezclado en presentación de harina para la elaboración de alimentos FES $(14,6)$ [7], sin embargo, los resultados son menores al rango más común de 15 a 29,8\% [9, 11, 20, 23, 24]. Esto mismo para el sauco $(23,36 \%)$ [5] y el tilo $(23,8 \%)$ [12]. Lo que se relaciona con la edad de las plantas debido al cambio metabólico que se presenta a medida que avanza la madurez de hojas y los tallos, ya que pueden alcanzar niveles de proteína de $35 \%$ en los primeros meses [25] pero luego disminuye.

Igualmente, se relaciona con las condiciones ambientales y la no aplicación de fertilizante químico en el banco forrajero, sin embargo en morera se ha observado proteína de $15,23 \%$ sin aplicación de nitrógeno con respecto a un 16,43\% PC cuando se ha fertilizado con $450 \mathrm{~kg}$ de N/ha/ año [26], lo que demuestra un leve aumento sin ser significativo, de modo que la fertilización no siempre favorece el contenido de proteína de las 
plantas, de modo que esto se relaciona más con las características edafoclimáticas específicas de la región y al comportamiento genético de la variedad [23] [27] [28] aunque también con la madurez del cultivo y tipo de cultivo por ejemplo en frutos la proteína no suele ser mayor al $1 \%$ [29] y en algunos pseudocereales como la quinoa está alrededor entre 16 y $20 \%$ [30].

\subsection{Extracto Etéreo (EE)}

Las varianzas de los resultados fueron homogéneos $(\mathrm{p}<0,05)$ y hubo diferencias significativas en todas las especies arbustivas ( $p$ $<0,05)$ con un mayor valor para tilo $(1,8 \pm 0,05)$, seguido de sauco $(1,4 \pm 0,05)$ y morera $(0,85 \pm 0,01)$. Este parámetro nutricional indica el contenido de grasa de un material arbustivo y de componentes como vitaminas liposolubles esteroles, resinas, ceras, pigmentos o aceites volátiles. Además, permite estimar el contenido calórico del material [31], triacilglicéridos de las semillas, galactolípidos y fosfolípidos de las hojas [11].

Todos los datos de EE se encuentran entre los esperados para pastos y forrajes tropicales $(0,56$ y $5,81 \%$ de la MS) [17]; sin embargo, se pueden encontrar valores superiores para sauco entre 1,9 y $5,2 \%$ [23], al igual que para tilo de 5,21\% [11] y morera de 2,1 - 2,46\% [8] [14] aunque puede llegar a $5,57 \%$ a las cuatro semanas de edad [21]. La variación entre las especies evaluadas se asocia con las moléculas de origen lipídico que abundan en el material vegetal, por ejemplo, en el sauco se reconoce la presencia de ácido palmítico [19], aunque es posible que estos valores no solo indiquen el contenido de grasa de los alimentos, sino que también incluyan sustancias fenólicas [16].

\subsection{Cenizas (CEN)}

Sauco obtuvo mayor contenido de cenizas $(10,07 \pm 0,01)$ seguido de morera $(8,6 \pm 0,05)$ y tilo $(1,8 \pm 0,05)(\mathrm{p}<0,05)$, valores inferiores a los observados en otro estudio con $11,8 \%$ en tilo, $20 \%$ en morera [11] y 9,81\% en sauco [9]. Las cenizas representan el contenido mineral de los alimentos menos el yodo y el selenio porque se volatilizan y su valor puede considerarse como un criterio útil para determinar la calidad de un alimento [31]. De modo que alto contenido de cenizas es deseable porque se atribuye mayor aporte de $\mathrm{Ca}$ [4]. Se ha encontrado en morera valores de $\mathrm{Ca} 3,73 \%, \mathrm{Mg}$ $0,40 \%$, Na 0,01\%, K 3,13\%, Fe 102,93ppm, Cu 7,99ppm, Zn 62,46ppm, P 0,58 \%, S 0,06\% y en sauco Ca de 1,9\%, Mg 0,61\%, Na 0,01\%, k $3,70 \%$, Fe 112,41ppm, Cu 6ppm, Zn 60,95ppm, P $0,62 \%$, S $0,25 \%$ [20].

El contenido de minerales en los forrajes se relaciona con las características químicas del suelo, fertilización y con la edad de las plantas [8]. Este valor toma importancia en la formulación y suministro de dietas por su influencia directa en la digestibilidad en relación a la forma en que se encuentren distribuidos dentro del alimento, sus interrelaciones y las fracciones orgánicas presentes en este que llevaran a suplir requerimientos en el organismo animal [14].

\subsection{Fibra cruda (FC\%)}

Los resultados tuvieron homogeneidad en sus varianzas $(p<0,05)$ y hubo diferencias significativas entre el aporte de FC de la morera $(18,97 \pm 0,01)$ y las especies arbustivas sauco $(17,8$ $\pm 0,05)$ y tilo $(17,92 \pm 0,18)$. La fibra representa las paredes celulares de los forrajes y se constituye por la celulosa y la hemicelulosa en mayor porcentaje, seguida de lignina, pectina, inulina, agar, quitina, gomas y silicatos, compuestos fenólicos, ácido fítico y otras moléculas antinutricionales presentes en muy pequeñas cantidades [32].

La concentración de fibra del forraje aumenta el consumo si no supera el valor restrictivo para cada especie mientras que altos aportes de energía lo disminuyen, de modo que con los resultados se infiere que sauco posiblemente presenta una mayor aceptación al consumo animal, basados en el contenido de FC de los forrajes [27]. Además, es un parámetro de los alimentos que influye sobre la tasa de degradación ruminal de los sustratos, adem toma importancia como porción orgánica de los alimentos difícil de digerir y selectiva para ciertas especies animales de acuerdo a su anatomía y fisiología digestiva [28]. 


\subsection{Fibra detergente neutra (FDN\%)}

El mayor contenido de FDN se observó en tilo $(37,8 \%)$, seguido de sauco $(29,9 \%)$ y de morera $(23,1 \%)$ con diferencias significativas únicamente para tilo y morera $(p<0,05)$. Los árboles forrajeros con bajos contenidos de FDN (20-35\%) presentan usualmente alta digestibilidad mientras que, si en la ración se incluye más del $22 \%$ de fibra, se perjudica la capacidad de consumo de alimento del animal, actividad de la flora microbial y por lo tanto la producción y calidad de la leche [11]. Lo que lleva a considerar a morera y sauco como los más digestibles. No obstante en sauco se puede encontrar FDN entre 19,4 y 42,8\% [33] [15], en tilo $19,44 \%$ [28] y en morera $40,3 \%$ [6].

\subsection{Fibra detergente acida (FDA\%)}

Hubo homogeneidad en las varianzas $(\mathrm{p}<$ $0,05)$ de los resultados obtenidos en el contenido de FDA entre los materiales evaluados con $47,3 \pm 0,05$ en tilo, $34,8 \pm 0,05 \%$ en sauco, $y$ $29,4 \pm 0,05 \%$ en morera $(p<0,05)$. La FDA en un forraje se refiere a las porciones de pared de las células constituida por celulosa y lignina y toma importancia al estar relacionada con la capacidad del rumiante para digerir el forraje de manera inversa ya que a medida que el porcentaje de FDA aumenta disminuye la digestibilidad del mismo [33].

$\mathrm{Su}$ contenido en forrajes varía, en sauco puede estar entre 15,8 y $38,7 \%$ [32][34][35] en tilo se ha observado $17,28 \%$ [15], y en morera entre el 17,4 a 24\% [24]. Con estos resultados se estima que la mejor digestibilidad la presenta morera, seguida de sauco y finalmente tilo.

\section{8 Área foliar $\left(\mathrm{cm}^{2}\right)$}

Hubo homogeneidad en las varianzas de los resultados de área foliar $(\mathrm{p}>0,05)$ y diferencias significativas $(p<0,05)($ Cuadro 2$)$. Esta variable es útil para caracterizar la dinámica y productividad de los ecosistemas forestales y permite entender la capacidad de las plantas para interceptar la luz [36], así como la reducción de estrés por calor durante periodos de radiación intensa, la estabilidad mecánica y a su vez el conocimiento frente al crecimiento y adaptación de la misma a los cambios climáticos [37, 38, 39, 40], además es reflejo de la fijación de carbono en las hojas y el funcionamiento de la maquinaria fotosintética de la planta, lo que permite determinar la especie con mejor adaptabilidad en sistemas de cultivo, que para el estudio corresponde a tilo, seguido de sauco y finalmente morera.

Tabla 2. Área foliar, producción de forraje verde y rendimiento de biomasa de las especies morera (Morus alba L.), tilo (Sambucus peruviana), sauco (Sambucus nigra L.)

\begin{tabular}{ccc}
\hline ARBUSTO & Área foliar $\left.\mathbf{( c m}^{\mathbf{2}}\right)$ & Forraje verde $\mathbf{( k g )}$ \\
\hline Sauco & $89,98 \pm 0,01$ & $1 \pm 0,04$ \\
Morera & $81,82 \pm 0,01$ & $1,73 \pm 0,05$ \\
Tilo & $131,37 \pm 0,01$ & $2,23 \pm 0,05$ \\
\hline
\end{tabular}

Letras diferentes indican diferencias significativas con un $\mathrm{p}<0,05$ por la prueba de tukey. \pm : error estándar

Lo anterior indica que el área foliar y la producción de forraje están directamente relacionados lo que resulta útil para predecir el rendimiento del cultivo en diferentes condiciones ambientales de modo que el índice de área foliar para el tilo fue mayor $(2,7)$ respecto a sauco $(1,4)$ y morera $(0,5)$, sin embargo al hacer la relación del área foliar entre de acuerdo al número de plantas por especie arbustiva la fijación de carbono fue más alta en sauco. Estos resultados inferiores a 3 muestran que se trata de un banco forrajero en fase de madurez ya que los valores más altos del índice de área foliar se manifiestan en el máximo crecimiento de las especies arbustivas como sucede cuando hay floración y posteriormente su valor disminuye como consecuencia de la senescencia de las hojas y perdida de vigorosidad del cultivo. 


\subsection{Producción de forraje verde (kg)}

Las varianzas de los datos de producción de forraje verde fueron homogéneos $(p>0,05)$ $\mathrm{y}$ hubo diferencias significativas entre las tres especies forrajeras $(\mathrm{p}<0,05)$. La producción de biomasa fue de $1,8 \pm 0,05 \mathrm{~kg} / \mathrm{m}^{2}$ para tilo, $0,66 \pm$ $0,05 \mathrm{~kg} / \mathrm{m}^{2}$ para morera y $0,38 \pm 0,05 \mathrm{~kg} / \mathrm{m}^{2}$ para sauco, donde cada uno estuvo ocupando un área aproximada de 2,6 $\mathrm{m}^{2} /$ planta. De modo que se estima una producción de forraje por poda de 2,5 ton / ha en morera, 6,9 ton / ha en tilo y 1,4 ton / ha en sauco cuando no se realiza fertilización en plantas de tres años en un banco forrajero. Estos resultados fueron mayores al evaluar morera $\left(1,73 ; 1,78\right.$ y $\left.1,57 \mathrm{~kg} / \mathrm{m}^{2}\right)$ y sauco $(2,90 ; 2,27$ y $\left.1,56 \mathrm{Kg} / \mathrm{m}^{2}\right)$ a los seis meses de siembra [2] lo que se atribuye a su estado de madurez, la fertilización y las condiciones de suelo.

\section{CONCLUSIONES}

La madurez del cultivo y la falta de fertilizante influyeron en el valor nutricional del forraje y en la producción de biomasa que no fue mayor de 2,5 ton / ha en morera, 6,9 ton / ha en tilo y 1,4 ton / ha en sauco por poda. Esto se debe a que la extracción de nutrientes es más alta que la incorporación por efecto de la hojarasca o por la actividad microbiana en el suelo en un banco forrajero sin fertilización, pero pese a esto la producción del forraje se mantuvo.

Las tres especies arbustivas tuvieron un contenido de FDN inferior a $38 \%$ y FC entre 17,8 - $18,9 \%$ de modo que se consideran materiales de buena digestibilidad, pero podas tardías pueden incrementar su valor como consecuencia de un aumento en el grosor de las paredes celulares y reducción en el contenido celular lo que a su influye en la calidad nutritiva del forraje.

El tilo presento mayor potencial productivo en comparación con morera y sauco por ello se considera un material potencial para ser incorporado en los sistemas ganaderos de la zona por presentar mayor índice de área foliar en el cultivo lo que se relaciona con su adaptabilidad en clima frío. Se concluye que los bancos forrajeros son una alternativa nutricional aceptable para el departamento de Boyacá porque en este arreglo las plantas toleran mejor las condiciones ambientales y logran mantener aportes de proteína a bajas tasas de fertilización, sin embargo, no se recomienda la incorporación de morera porque su potencial lo expresa mejor en zonas templadas, aunque puede crecer a diferentes altitudes.

\section{AGRADECIMIENTOS}

Los autores manifiestan agradecimientos al grupo de investigación en Bioquímica y Nutrición Animal (GIBNA) por su colaboración en el análisis de las muestras en el laboratorio de nutrición animal de la Universidad Pedagógica y Tecnológica de Colombia (UPTC).

\section{REFERENCIAS}

G. M. Blanco, D. R. Chamorro, L. C. Arreaza, A. M. Rey. "Evaluación nutricional del ensilaje de Sambucus peruviana, Acacia decurrens y Avena sativa". Revista Corpoica. 6(2):81-85. 2005. Doi: https://doi.org/10.21930/rcta.vol6_num2_art:52

C. Rodríguez-Molano, D. Fonseca-López D. Nutrición animal, lípidos y fitoestrógenos. Tunja: Editorial Uptc. 2018. ISBN: 9789586602747.

F. Espinoza, C. Araque, L. León, H. Quintana, E. Perdomo. "Efecto del banco de proteína sobre la utilización del pasto estrella (Cynodon lemfuensis) en pastoreo con ovinos". Zootecnia Tropical. 19(1):307-318. 2001. Recuperado de: http://www.bioline.org.br/request?zt01038

G. J. Martín, Y. Noda, G. Pentón, D. E. García, F. García, E. González, F. Ojeda, M. Milera, O. López, J. Ly, L. Leiva y J. Arece. "La morera (Morus alba, Linn.): una especie de interés para la alimentación animal". Pastos $y$ Forrajes. 30(5):1-1. 2007. Recuperado de: http://scielo.sld.cu/scielo.php?script=sci arttext\&pid=S0864-03942007000500001

C. J. Jaramillo-Isaza. "Evaluación agronómica de Morus alba L y Sambucus Nigra L y su utilización en alimentación de rumiantes y monogástricos". Revista de investigación. 6(2):189-197. 2006. Recuperado de: http://www.redalyc.org/ pdf/952/95260206.pdf 
M.M. Bonilla-Morales, A.C. Aguirre-Morales, H. López-Toscano. "Diversidad y conservación de Catasetum (Orchidaceae: Catasetinae) en el Departamento de Santander, Colombia". Revista Ciencia en Desarrollo. 7(2) 57-65. 2016. Doi: https://doi.org/10.19053/01217488. v7.n2.2016.3925

D. Fonseca-López, C.E. Rodríguez-Molano, M. García-Parra. "El compostaje, una biotécnica para el tratamiento de residuos". RevistaInfometric@ - Serie Ingeniería, Básicas y Agrícolas. 1(1):1523. 2018.

B. Mostacedo, T. Fredericksen. "Manual de Métodos Básicos de Muestreo y Análisis en Ecología Vegetal”. Ed Santa Cruz Bolivia. 2000.

D. Fonseca-López, G. Saavedra-Montañéz, C. Rodríguez-Molano. "Elaboración de un alimento para ganado bovino a base de zanahoria (Daucus carota L.) mediante fermentación en estado sólido como una alternativa ecoeficiente". Revista Colombiana De Ciencias Hortícolas. 12(1):175182. 2018. Doi: https://doi.org/10.17584/ rcch.2018v12i1.7416

F. R. Garcés, C. A. Forcelini. "Peso de hojas como herramienta para estimar el área foliar en soya". Ciencia y Tecnología. 4(1): 13-18. 2011. Doi: https://doi:10.18779/cyt.v4i1.156

C. Boschini, C. F. Vargas. "Rendimiento y calidad de la morera (Morus alba) fertilizada con nitrógeno, fósforo y potasio". Agronomía mesoamericana. 20(2): 285-296. 2009. Recuperado de: http:// www.mag.go.cr/rev_meso/v20n2_285.pdf

J. E. Apráez, J. M. Delgado, J. P. Narváez. "Composición nutricional, degradación in vitro y potencial de producción de gas, de herbáceas, arbóreas y arbustivas encontradas en el trópico alto de Nariño". Livestock Research for Rural Development, 24 (3): 1-17. 2019. Recuperado de: http://www.lrrd.org/lrrd24/3/apra24044.htm

T. Carvajal-Salcedo, A. Cuesta-Peralta. "Conservación y composición nutricional del follaje de sauco (Sambucus nigra)". Pastos y Forrajes. 39 (2):125-132. 2016. Recuperado de: http://scielo.sld.cu/scielo.php?script=sci arttext\&pid=S0864-03942016000200007
E. B. Trabi, X. Yuan, J. Li, Z. Dong, A. A. Shah, T. Shao. "Effect of glucose and lactic acid bacteria on the fermentation quality, chemical compositions and in vitro digestibility of mulberry (Morus Alba) leaf silage". Pakistan Journal of Zoology. 49 (6): 2271-2277. 2017. Doi: http://dx.doi. org/10.17582/journal.pjz/2017.49.6.2271.2277

J. Condo. "Tema: Estudio de Factibilidad de un banco de Proteína a partir de Morera (Morus alba), Tilo (Sambucus peruviana), Leucaena, (Leucaena leucocephala), Cañaro (Erythirina edulis) en el Cantón Paute sector Cachiacu". Tesis de pregrado, Facultad de Ciencias Agropecuarias, Universidad de Cuenca, 2012.

Y. Noda, G. Martín, R. Machado. "Rendimiento agronómico de la morera por efecto de diferentes alturas y frecuencias de corte". Pastos y Forrajes. 30 (3): 327-339. 2007. Recuperado de: http://scielo.sld.cu/scielo.php?script=sci arttext\&pid=S0864-03942007000300004

A. Alpízar. "Efecto de la suplementación con Morus alba Linn en la ceba de ovinos Pelibuey en estabulación". Tesis de maestría, Estación experimental de pastos y forrajes Indio Hatuey, Universidad de Matanzas Camilo Cienfuegos, 2014.

D. I. Hurtado, S. Nocua, W. Narváez, J. E. Vargas. "Valor nutricional de la morera (Morus sp.), matarratón (Gliricidia sepium), pasto india (Panicum máximum) y arboloco (Montanoa quadrangularis) en la alimentación de cuyes (Cavia porcellus)". Veterinaria y zootecnia. 6(1):56-65. 2012. http://vip.ucaldas.edu.co/ vetzootec/ downloads/v6n1a06.pdf

S. Srivastava, R. Kapoor, A. Thathola, R. P. Srivastavan. Nutritional quality of leaves of some genotypes of mulberry (Morus alba), International Journal of Food Sciences and Nutrition. 57 (5-6): 305-313. 2006. Doi: https://10.1080/09637480600801837

Y. M. Jaramillo, J. N. Jiménez. "Evaluación nutricional de tres especies de árboles forrajeros en la alimentación de vacas Holstein en el trópico alto de Nariño". Tesis de pregrado, Facultad de Ciencias Pecuarias, Programa de zootecnia, Universidad de Nariño, 2000. 
N. Miles, L. Thurtell. S. Riekert. "Quality of Kikuyu herbage from pastures in the Eastern Cape coastal belt of South Africa". South African Journal of Animal Science. 30(1): 85-86. 2000. Doi: http://dx.doi.org/10.4314/sajas.v30i4.3921

O. Sepulveda-Delgado, Z-E. Suarez-Aguilar, M. Patarrollo-Mesa, L.C. Canaria-Camargo, S. Bautista-Díaz. "Estudio del comportamiento e impacto de la climatología sobre el cultivo de la papa y del pasto en la región central de Boyacá empleando los sistemas dinámicos". Revista Ciencia en Desarrollo. 6(2): 215-224.2015. Doi: https://doi.org/10.19053/01217488.3792

R. A. Al-Kirshi, A. Alimon, I. Zulkifli, S. Atefeh, M. W. Zahari, M. Ivan. "Nutrient digestibility of mulberry leaves (Morus alba)". Italian Journal of Animal Science. 12 (36): 219-221. 2013. Doi: https://doi.org/10.4081/ijas.2013.e36

M.C. Blanco, M. L. Sierra. "Caracterización bromatológica y evaluación de diferentes niveles de inclusión de morera (Morus alba L.) y sauco (Sambucus nigra L.), en la alimentación de conejos en ceba". Tesis de Pregrado, Facultad de Zootecnia, Universidad de la Salle, 2005.

B. M. Grajales, M. M. Botero, J. F. Ramírez. "Características, manejo, usos y beneficios del saúco (Sambucus nigra L.) con énfasis en su implementación en sistemas silvopastoriles del Trópico Alto". Revista de Investigación Agraria y Ambiental. 6(1): 155-168. 2015. Doi: https://doi. org/10.22490/21456453.1271

J. A. Elizondo. "Respuesta de la morera (Morus alba) a niveles crecientes de nitrógeno orgánico". Pastos y forrajes. 33(3): 1-12. 2010. Recuperado de: $\quad$ http://scielo.sld.cu/scielo.php?script $=$ sci arttext\&pid $=$ S0864-03942010000300004

Y. Noda, G. J. Martín. "Influencia de la densidad de plantación y la fertilización nitrogenada en el rendimiento de Morus alba var. Tigreada". Pastos y Forrajes. 37(3): 291-297. 2014. Disponible en: http://scielo.sld.cu/scielo.php?script=sci_ arttext\&pid=S0864-03942014000300006

D. Fonseca-López, \& L. Borras-Sandoval. "Evaluación del efecto de la papa fresca incluida en un alimento para vacas Holstein sobre la producción y la calidad de la leche". Ciencia y Agricultura. 11(1), 55-65. 2014. Doi: https://doi. org/10.19053/01228420.3488

G.M. Cortés-Díaz, G.A. Prieto-Suárez, W.E. Rozo-Nuñez. "Caracterización bromatológica y fisicoquímica de la uchuva (Physalis peruviana L.) y su posible aplicación como alimento nutracéutico". Revista Ciencia en Desarrollo. 6(1): 87-97. 2015. Doi: https://doi. org/10.19053/01217488.3653

M. García-Parra, N. Plazas-Leguizamón, D. Fonseca-López. "Potencial nutricional de semillas de quinua (chenopodium quinua willd) en colombia: una revisión”. Revista Infometric@ - Serie Ingeniería, Básicas y Agrícolas. 1(1): 8593. 2018

V. Dardon, M. Duran. "Cuantificación espectrofotométrica de taninos y análisis bromatológico proximal de cuatro diferentes mezclas de forrajes a base de gramíneas y leguminosas". Tesis de Pregrado, Facultad de química y farmacia, Universidad de el Salvador, 2011.

F. L. García. "Evaluación agronómica de la morera (Morus alba cv. cubana) en un suelo ferralítico rojo típico". Tesis de Maestría, Universidad de Matanzas Camilo Cienfuegos, 2004.

C. A. Rossi. "Composición Florística y caracterización nutricional de las especies forrajeras en el sistema silvopastoril del delta del Paraná". Tesis de Doctorado, Facultad de Ciencias Agropecuarias, Universidad Nacional De Córdoba, 2013.

C. A. Cárdenas, C. Rocha, J. Mora. "Productividad y preferencia de forraje de vacas lecheras pastoreando un sistema silvopastoril intensivo de la zona alto Andina de Roncesvalles, Tolima". Revista Colombiana de Ciencia Animal. 4(1):2935. 2011. Disponible en: http://revistas.ut.edu.co/ index.php/ciencianimal/article/view/140/139

H. Millán, F. Moreno. "Evaluación agronómica de arbóreas mutipropósito en la sabana de Bogotá". Tesis de pregrado, Universidad Nacional de Colombia, 2005. 
C. A. Aguirre-Salado, J. R. Valdez-Lazalde, G. Ángeles-Pérez, H. M. De los Santos-Posadas, A. I. Aguirre-Salado. "Mapeo del índice de área foliar y cobertura arbórea mendiante fotografía hemisféricaydatos SPOT5HRG:regresiónyk-nn". Agrociencia. 45(1):105-119. 2011. Disponible en: http://www.scielo.org.mx/scielo.php?script=sci arttext\&pid=S1405-31952011000100010

M. Westoby, D. S. Falster, A. T. Moles, P. A. Vesk, I.J Wright. "Plant ecological strategies: some leading dimensions of variation between species". Annual Review of Ecology and Systematics. 9(1):125-159. 2002. Disponible en: http://www.medellin.unal.edu.co/ poboyca/ documentos/documentos1/Nivelaci\%F3n $\% 20$ Ecolog\%EDa\%20Forestal-Polania/Westoby\%20 et $\% 20$ al $\% 202002$.pdf
H. Pobres, C. Remkes. "Leaf area ratio and net assimilation rate of 24 wild species differing in relative growth rate". Oecologia. 83(4):553559. 1990. Disponible en: www.jstor.org/ stable/4219374

J. M. Buswell, A. T. Moles, S. Hartley. "Is rapid evolution common in introduced plant species?". Journal of Ecology. 99:214-224.2011.Doi: https:// doi.org/10.1111/j.1365-2745.2010.01759.x

Y. Álvarez, E. Álvarez, J. Cano, D. Suescun. "Modelo matemático para estimar área foliar en árboles del bosque tropical seco en el caribe colombiano". Revista del Instituto de Investigaciones Tropicales. 7: 69-79. 2012. Disponible en: http://revistas.unimagdalena.edu. co/index.php/intropica/article/view/165 